\title{
A lightweight CNN model and its application in intelligent practical teaching evaluation
}

\author{
$Y i \mathrm{He}^{*}$ and Tianli $\mathrm{Li}$ \\ City Institute, Dalian University of Technology, Dalian 116600, China
}

\begin{abstract}
Keywords: CNN, Lightweight, Parameter redundancy, Practical teaching, Intelligent evaluation.

Abstract. In this paper, we propose a lightweight CNN model. Firstly, we standardize the existing CNN model structure based on the minimum computing unit, and second we apply a parameter control solution to solve the problem of parameter redundancy in the model. At last we build a lightweight nonaligned CNN model. The experimental results show that the model parameters can be reduced by more than $50 \%$ when the test error is almost the same. Through deep learning, the proposed model is applied to the practical teaching system to achieve the intelligent evaluation effect of the practical teaching process, while improve the quality and efficiency of teaching.
\end{abstract}

\section{Introduction}

Convolutional neural network (CNN) model [1] has significant advantages in feature extraction, and is widely used in computer vision fields such as image classification [2], target detection [3], style recognition [4], scene segmentation [5] . Compared with the traditional model of manual extraction of visual features [6], there are many kinds of network structures. These structures can be divided into three types: single chain CNN [7, 8], multi branch misaligned CNN [9-11] and single chain CNN with jump connection. With the deeper and deeper framework of the network model, more and more training parameters are needed, which leads to the performance of the model heavily dependent on computing resources. Therefore, the research on reducing the parameter redundancy of convolutional neural network is particularly important.

In this paper the structure of nonaligned network proposed abandons the model structure based on layer, and takes the minimum computing unit as the unit and the minimum computing unit as the node of network model. Each minimum computing unit represents a partition benchmark. Therefore, there is no alignment form of CNN model in nonaligned network, which greatly reduces the redundancy of parameters. While the redundancy of the parameters is greatly reduced, the performance of the model is also guaranteed to be free of great loss, which requires the operation of feature fusion [12]. The proposed model applies cascading operation to combine the features learned by different minimum computing unit nodes, make full use of the information hidden in the model, increase the diversity of node

*Corresponding author: Heyi517@dlut.edu.cn 
input, and make the model obtain greater representation ability. The experimental results show that compared with DenseNet40, this method can reduce the model parameters by more than $50 \%$ on the premise that the test error is less than $1 \%$.

Nowadays there are many researches on "artificial intelligence + education"[13, 14]. Through deep learning based on the application of the proposed model, we can realize the intelligent evaluation of the practical teaching operation process, and finally according to the operation progress and accuracy of the students' completion of the practical task, we give the final score of the practical course by the weight formula in the teaching. It makes the evaluation of practical courses more efficient, optimizes the process of teachers' management, supervision and evaluation of teaching, improves the quality of formative assessment of practical teaching, and improves the construction of information and intelligence of practical teaching.

\section{LCNN model based on nonaligned network}

\subsection{Design of LCNN model}

The network structure of Lightweight Convolutional Neural Network (LCNN) is composed of two parts: the minimum node set of computing unit (recorded as $N$ ) and the set of edges connecting two nodes (recorded as $E$ ). Therefore, LCNN can be expressed as:

$$
L C N N=(N, E)
$$

For the minimum cell node set $N=\left\{N_{i} \mid i=1, \ldots, m\right\}$, the nodes can be used as a separate convolution operation or combined into multiple convolution operations, which ensures the diversity of convolution operations that can be expressed by the minimum computing unit node set. For a set of edges connecting two nodes $E=\left\{E_{i j} \mid 1<i<j \leq m\right\}$, it is specified that they cannot have a connection to themselves, that is, the non-aligned network structure does not contain rings. In order to ensure $N$ that any node in the node set of the minimum computing unit participates in feature learning, the set of values $i, j$ in the edge set $E$ should meet the requirements $I \cup J=M$, where $M$ is the set of values $i$ that can be obtained in the set $N$.

In order to ensure the lightweight of LCNN, we stipulate to reorder the minimum computing unit node set $N$ according to the number $\alpha$ of nodes with the same longest path and reassign it to group $b$ :

$$
N=\left\{N_{k b} \mid k=1, \ldots, b ; i=1, \ldots, a\right\},(m=a \times b)
$$

There is no connection between nodes of the same group with the longest path constraint. If the nodes of different groups are adjacent to each other, the corresponding nodes (the same) are connected. If the groups are not adjacent, the edges in the case of full connection of the nodes of the smallest computing unit are discarded with a certain probability value of discarding the connection. Therefore, the edge set $E$ is refined as: 


$$
\begin{aligned}
& E=\left\{E_{i j} \mid i \in\{(1,1),(1,2), \ldots,(b, a)\}\right. \\
& j \in\{(i+1,1),(i+1,2) \ldots,(i+1, a)\} \cup \alpha * \operatorname{drop}\{(i+2,1), \ldots,(b, a)\}\},
\end{aligned}
$$

where $\alpha$ is the given probability value of disconnection.

\subsection{Experimental environment and data}

Experimental environment: all experiments in this paper are implemented in the single CPU core $(2.40 \mathrm{GHz})$ of Intel Xeon e5-2640 server with $160 \mathrm{~GB}$ ram, and the operating system is CentOS 7.4.1708. Two NVIDIA K40 GPUs are used for CNN computing.

Cifar-10 data set [15]: this data set is composed of 10 kinds of color natural images with $32 * 32$ pixels. Its training set and test set contain 50000 and 10000 images respectively. For data enhancement, we use the standard data enhancement scheme which is widely used in the dataset. For the preprocessing stage, we use the mean and variance of the image to standardize the data. For each round of model training, we use all 50000 training images and report test errors at the end of training.

In this experiment, a network model of LCNN is designed: the combination of $0.3,0.5$ and 0.7 is selected to correspond to the probability value $\alpha$ of disconnection used by the three blocks of LCNN. The experimental results are shown in Table 1. According to the experimental results in Table 1, the number of parameters used in the LCNN model is reduced by more than $50 \%$ compared with DenseNet models, while the test error rate is only reduced by $0.82 \%$.

Table 1. Experimental results of LCNN and other models.

\begin{tabular}{|c|c|c|c|}
\hline Model name & $\begin{array}{c}\text { Model depth / } \\
\text { Longest path }\end{array}$ & Model parameter & $\begin{array}{c}\text { Test error rate (\%) } \\
\text { Cifar10 }\end{array}$ \\
\hline ResNet & 110 & 1727891 & 5.97 \\
\hline IGC & 38 & 2432646 & 5.70 \\
\hline DenseNet & 40 & 1019672 & 5.69 \\
\hline $\begin{array}{c}\text { LCNN } \\
(\alpha 1,2,3=0.3,0.5,0.7)\end{array}$ & 40 & 504251 & 6.51 \\
\hline
\end{tabular}

\section{The application of LCNN in intelligent practical teaching evaluation}

Because of the strong operability and process of experimental teaching, if the operation process is stored, analyzed and evaluated by video recording, the amount of data, flow and workload are large, and the efficiency of teaching evaluation is low. We can apply the LCNN model for deep learning to realize the intelligent evaluation of the progress and accuracy of the practical teaching operation process. The process is as follows:

(1) A practice process is divided into several steps, and the result renderings of correct practice operation in each step are one class, and one class includes the result renderings of correct practice operation in several perspectives.

(2) During the actual operation of the students, the system will take practice effect picture at the designated completion time of each step and pass them to the model. Through the analysis and discrimination of the model, it will give the probability that the picture belongs to each category, that is, the probability of correct effect picture of each step. The 
system will evaluate the operation of this step according to the highest probability value, for example, the picture taken by the system at the end time of the third step will pass through the LCNN model, and it can be concluded that the highest probability class of the picture belongs to the second class, that is, the second step effect picture, which can evaluate the slow progress of the student's practical operation. Otherwise, if it can be concluded that the highest probability class of the picture belongs to the fourth class, that is, the fourth step effect picture, it can be concluded that the student's practical operation is skilled and the progress is fast.

(3) When building the category, one will be an incorrect category, which provides the basis for judging the accuracy of students' operation.

(4) The system will give the score of the final practical task completion effect according to the progress and accuracy of the students' operation, and finally according to the weight formula in the teaching.

The diagram of the intelligent evaluation process of practical teaching is as follow Figure 1.

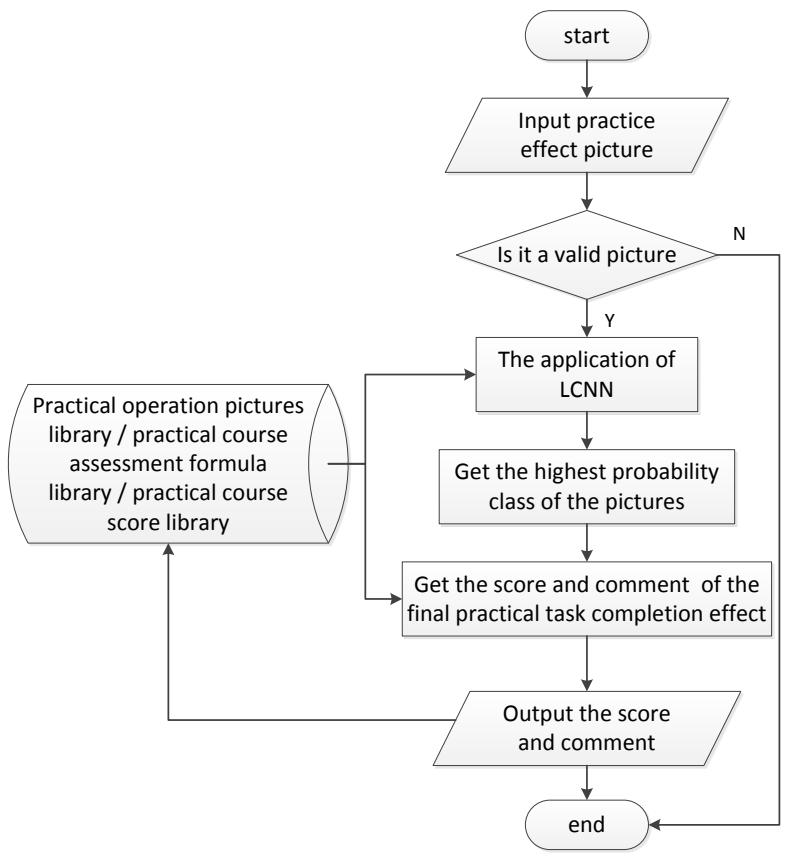

Fig. 1. The diagram of the intelligent evaluation process.

\section{Conclusion}

A LCNN model based on nonaligned network is proposed in this paper. As the smallest computing unit of the network node, the model can be combined, and the adjacent matrix of sparse network can be generated by a certain probability value of discarding connection, which makes the model lightweight. It can effectively reduce the number of parameters used in CNN model. We use LCNN model for deep learning and by analyzing the progress of students' operation and the accuracy of completing practical tasks, we realize the intelligent evaluation of the operation process of practical tasks, and improve the formative assessment mechanism of practical teaching. At the same time, it release a lot of time and energy of teachers, ensure the personalized teaching and guidance of teachers to students, improve the efficiency of teaching, and achieve the purpose of accurate practical teaching. 
This research is supported by the Liaoning Province 13th Five-Year Education Science Planning Project: Research on "Internet + AI" precise practice teaching mode (No. JG18DB092)

\section{References}

1. J. Gu, Z. Wang, J. Kuen, Recent advances in convolutional neural networks. (2015) arXiv:1512.07108.

2. D.Y. Zhang, Y.X. Chang, L.G. Zhang, SAT-CNN: convolutional neural network framew-ork for remote sensing image classification, Journal of Chinese Computer Systems. 39(4) (2018) 859-864.

3. J. Redmon, S. Divvala, R. Girshick, You Only Look Once: Unified, Real-Time Object Detection, Proceedings of the IEEE Conference on Computer Vision and Pattern Recognition. (2016) 779-788.

4. Z.H. Hu, Y.Y. Yu, Application of deep convolution network in music style recognition, Journal of Chinese Computer Systems. 39(9) (2018) 1932-1936.

5. H. Noh, S. Hong, B. Han, Learning Deconvolution Network for Semantic Segmentation, Proceedings of the IEEE International Conference on Computer Vision. (2015) 1520-1528.

6. O.A.B. Penatti, F.B. Silva, E. Valle, Visual word spatial arrangement for image retrieval and classification, Pattern Recognition. 47(2) (2014) 705-720.

7. Y. Lecun, L. Bottou, Y. Bengio, Gradient-based learning applied to document recognition, Proceedings of the IEEE. 86(11) (1998) 2278-2324.

8. C. Szegedy, W. Liu, Y. Jia, Going deeper with convolutions, (2014) arXiv:1409.4842,.

9. O. Russakovsky, J. Deng, H. Su, ImageNet Large Scale Visual Recognition Challenge, International Journal of Computer Vision. 115(3) (2015) 211-252.

10. G. Larsson, M. Maire, G. Shakhnarovich, Fractalnet: Ultra-deep neural networks without residuals. (2016) arXiv:1605.07648,.

11. T. Zhang, G.J. Qi, B. Xiao, Interleaved Group Convolutions. Proceedings of the IEEE Conference on Computer Vision and Pattern Recognition. (2017) 4383-4392.

12. R.K. Srivastava, K. Greff, J. Schmidhuber, Training very deep networks. Proceedings of the Conference on Advances in Neural Information Processing Systems. (2015) 2368-2376.

13. D. Alexey, B. Alexander, S. Tatyana, S. Natalya, Big Data and Artificial Intelligence for Digital Humanities: An International Master Program via Trans-Eurasian Universities Network, Procedia Computer Science, 101 (2016) 449-451.

14. B. Zhang, Z.H. Fang, H. YE, The Cultivation Pattern and Construction Path of “Artificial Intelligence Educational Talent” in Emerging Engineering Era, Modern Educational Technology. 8 (2019) 113-119.

15. Krizhevsky, Alex, Learning Multiple Layers of Features from Tiny Images. First ed., Master's thesis, University of Toronto, 2009. 